\title{
On Planning of FTTH Access Networks with and without Redundancy
}

\author{
M. Tahir Riaz, Gustav H. Haraldsson, Jose G. Lopaz, Jens M. Pedersen, \\ and Ole B. Madsen
}

Aalborg University, Networking and Security Section, Fredrik Bajers vej 5, 9220 Aalborg, Denmark

tahir@es.aau.dk, gustav.haraldsson@gmail.com, jgl@es.aau.dk, jens@es.aau.dk, obm@es.aau.dk

\begin{abstract}
This paper presents a planning analysis of FTTH access network with and without redundancy. Traditionally, access networks are planned only without redundancy, which is mainly due to lowering the cost of deployment. As fiber optics provide a huge amount of capacity, more and more services are being offered on a single fiber connection. Therefore, as a single point of failure in fiber connection can cause multiple service deprivation, redundancy is very crucial. In this work, an automated planning model was used to test different scenarios of implementation. Cost estimation is presented in terms of digging and amount of fiber used. Three topologies, including the traditional one "tree topology", were tested with a combination of various passive optical technologies.
\end{abstract}

Keywords: FTTH, Network Planning, Future Networks, Reliability, Broadband, ICT infrastructure, Modeling, Passive Optical Networks.

\section{Introduction}

Data communication over the Internet has been exponentially growing since the 90's. The Plain Old Telephone System (POTS) network has been great in meeting the demands of increasing bandwidth (with Integrated Service Digital Network (ISDN), asymmetrical digital subscriber line (ADSL), Symmetric digital subscriber line (SDSL), etc.) demands over its copper network. However the POTS network is already being pushed to its limit in trying to offer more bandwidth with new technology over the coppers. The downside is that the user has to be closer and closer to the connecting equipment.

Today more and more services are being introduced through the copper based network, not only the Internet, therefore making it an all-purpose network. These services (VoIP, VoD, other Internet applications) are demanding a lot of bandwidth, therefore it is apparent that though the current network media holds, it is becoming exhausted and a new type of media in which to use this all purpose network, is required. Optical fiber is one possible successor in planning a new installation, upgrading or expansion of, and to, an all purpose network. Optical fiber offers new 
possibilities compared to the older medium of copper based communication systems. One of these possibilities is Fiber To The Home (FTTH), where the complete network infrastructure is fiber, from the supplier all the way to the Network Termination (NT) - end user. By doing so the NT has a greater amount of capacity available.

All NTs are thus now more than ever becoming reliant on the reliability of an allpurpose network, since all these services are now being transferred to this all it. However, today's network planning has mainly focused on the upper levels of the network when creating redundancies, rather than planning the access networks with non redundant topologies. But as NTs in the access network are relying on the network, some research is needed to offer redundancy to the access network without introducing too much extra cost.

This paper contributes with an analysis of implementing FTTH with redundancy at the infrastructure level, which includes digging and amount of fiber optic used. The paper also introduces a new topology, namely eartree topology, which is seen as a compromise for lowering the cost of infrastructure, hence still providing good redundancy [1].

The structure for the rest of the paper is as follows: Section 2 provides terms and definitions used in the paper. Section 3 presents the planning method. Section 4 presents different topologies for the FTTH access network. Section 5 presents a case study where the area of Hals (rural area in Denmark) was chosen as a test subject. Section 6 lists phases and implementation scenarios with achieved results. And finally Section 7 provides a conclusion to the work.

\section{Terms and Definitions}

Some terms and definitions are used throughout the paper in order to explain different aspects of the network. The Swedish ICT commission [2] has standardized some terms used in planning a fiber network. In order to clarify these terms they are explained here as they are used throughout the paper.

\subsection{Network Architecture}

Figure 1 shows how the network architecture is a set up of 3 network layers and is as follows:

Main network: The main network, sometimes called the backbone network, is the highest level in the municipality's network hierarchy. This layer connects the municipality to the external network with links going out of the municipality and also down to the distribution network.

Distribution network: The distribution network consists of distribution nodes and lines, which connect the access network. In this paper the distribution network is the municipal network.

Access network: The access network is a network of Network Termination points (NTs) connected to the distribution node. NTs nodes are households, apartment buildings, business and government places. This paper will not distinguish between them. 


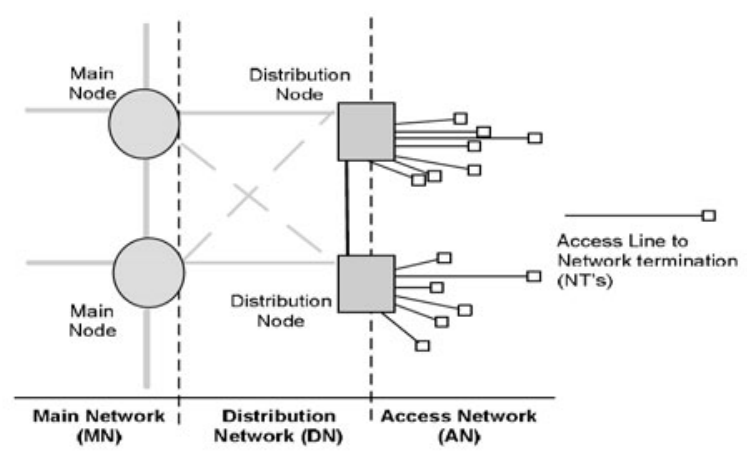

Fig. 1. Network architecture hierarchy

\section{Planning Method}

\subsection{GIS System}

A map can be used to represent real things that are in the real world; for example, roads, rivers or buildings.. This map information can be stored in a digital form in a system like GIS. As GIS data can store roads, rivers, bridges and other structures in a digital format, it is ideal for network planners to use it. Network planning with GIS data makes it easier to locate possible places through population density for Central Offices (COs) etc., as well as placements of pipes and lines for the communication infrastructure.

\subsection{Automated Network Planning}

Manual planning has a lot of drawbacks, i.e. it takes more time, costs more, and mistakes are easily made. If an access network is planned automatically it can reduce the degree of problems and drawbacks that can be observed in a manual process of planning an access network. To automate the planning process, GIS data and a set of algorithms were used to make the planning process more automatic. Network objects are modeled as nodes and links. The network model contains logical information such as connectivity relationships among nodes and links, directions of links or segments, and costs of nodes and links. With logical network information, it is possible to analyze a network.

The automated planning method consists of several steps, which are carried out in an iterated manner. In the first steps, the finding of potential traces for placing fibers and normalizing GIS data for traces are performed. When the GIS data is normalized, the parameters are set and different algorithms are applied to generate the plans. The flow diagram of the planning method is depicted in Figure 4. The key elements of the automated planning model are the trace normalization and planning algorithms. In the trace normalization, the digital road network is simplified by reducing the number segments. When the trace network is normalized and the parameters set, the trace network is processed further by a set of graph algorithms. In this study: spanning tree, shortest path, and various modified path algorithms were used. The algorithms 
perform calculations for the laying of fiber cables and ducts based on the specified parameters. The planning algorithm selects the traces for fiber, which gives optimal paths (i.e. shortest or cheapest).

\section{FTTH Access Network Topologies}

The basic idea behind network topologies is to explain a network and is determined by the configuration between the nodes in a network. While some network topologies show simple means in connecting a network, other network topologies are more focused on offering redundancy and availability in a network.

\subsection{Topologies}

Today access networks are planned in a cheap manner and without redundancy. This means that in most case with a tree topology, as seen in Figure 2a, it branches out to each NT in the shortest and cheapest way possible. It is clear that this type of network topology offers no redundancy, so in the case of a line break near the root of the tree, it would cause several NTs to be affected. A topology designed for the access network might be able to offer redundancy by cutting projected cost. Figure $2 \mathrm{~b}$ shows the ear topology and its design cut extra costs by reducing the extra digging needed [3]. Here two fibers are planned for each NT from two different distribution nodes, creating a closed loop to each NT, and therefore, redundancy.

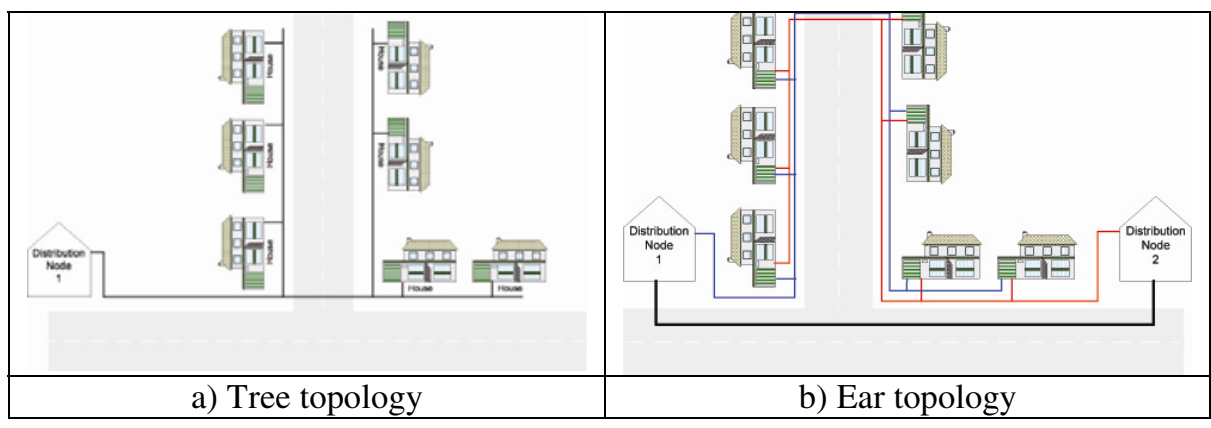

Fig. 2. Access network topologies

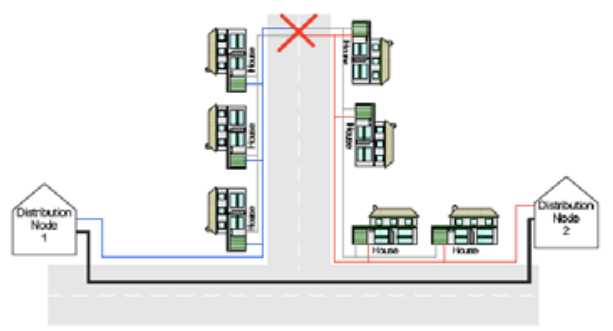

Fig. 3. Ear topology with single point of failure 
The digging is then done by placing the fibers in the same trench but coming from opposite directions reducing the cost of digging with the reuse of trenches. There is a golden rule to be used with the ear topology which is: Trenches can never be shared in the same direction to - or within - the same districts. In the case of a failure the NTs can switch to the active line, keeping the connection available, as can be seen in Figure 3. A previous study has shown that by reusing trenches extra digging costs were cut and the amount of extra fiber was tripled using ear topology [4]. That study however, only used the manual planning approach.

\subsection{New Topology, Splicing Points and Districts}

A new topology is also introduced, which is the combination of two previously mentioned topologies - the tree topology and the ear topology. The new topology referred

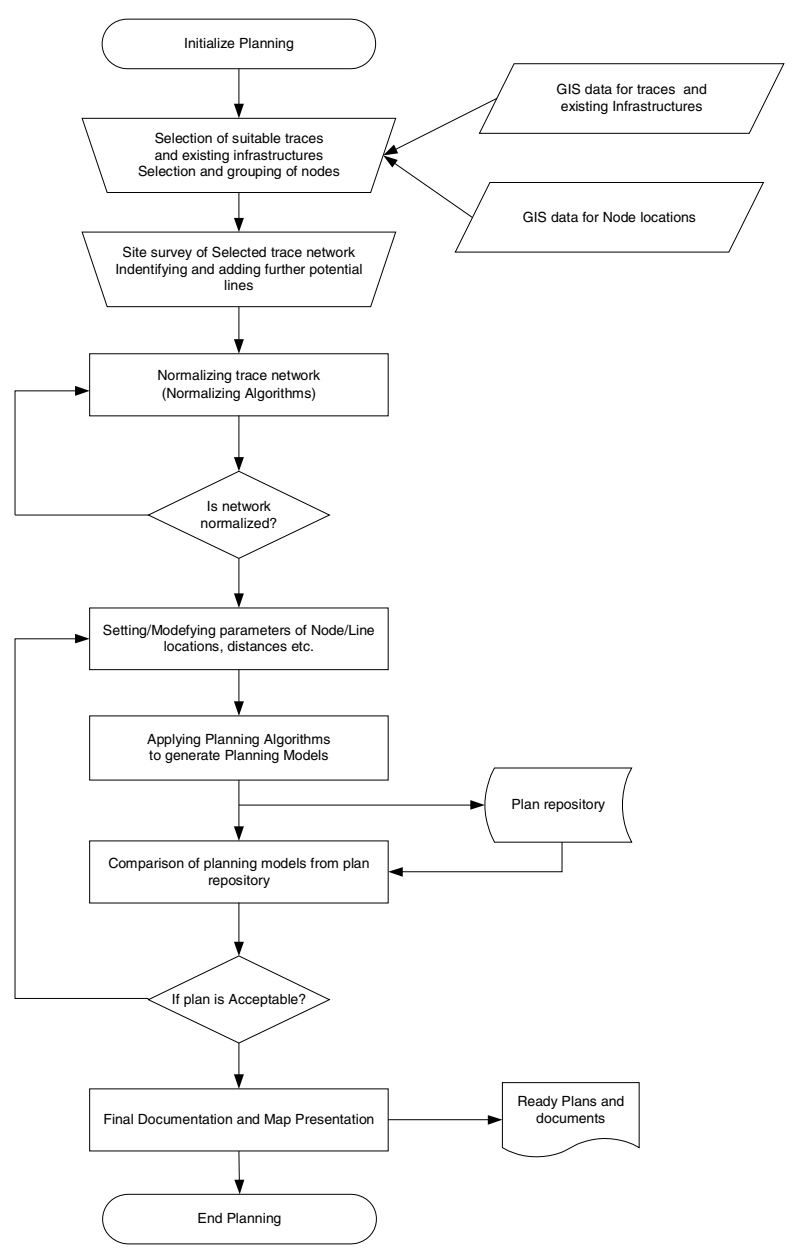

Fig. 4. The flow diagram of automated planning method 
hereafter as the "eartree topology" utilizes both ideas, that of the ear topology from the DN to splicing points (SPs) and the tree topology from the SP to NTs. The eartree topology can be seen in Figure 7a and Figure 7b.

That means that each SP would have two separate fibers from two separate DNs causing the SP to be redundant. From the SP to the NTs the tree topology is used. So the main amount of extra fiber shown in the previous study [4] of the ear topology is addressed. It is obviously not as redundant as the ear topology, but that loss of redundancy is minimized because the most important part of the way is redundant.

Districts are created in correlation with the SPs and by doing so we can simplify the automated network design process by linking the NTs within a distance to a SP. It is also possible to view the affect that different sizes of district have on the cost of the eartree topology. As single fiber cables cost relativity more per NT then a multi strand cable does, it is also interesting to see what affect decreasing the size of each district, and having the SPs closer to the NTs, has. By finding the right balance we might be able to decrease fiber cost while make the design more redundant at the same time.

\subsection{Home-Run and Passive Optical Network (PON)}

The home-run (HR) architecture is a network that can be realized with a pair of fibers that runs from the $\mathrm{CO}$ to each NT, due to this reason the home-run architecture is also called point-to-point architecture [5]. This architecture uses a larger amount of fibers, that all demand separate termination at each end. Figure 5a shows the home-run architecture. The main drawback of this type of architecture is the fiber cost [6].

A PON is a point to multi-point network over a passive fiber plant comprising fibers, splitters and an active equipment at the end [5]. The PON architecture is used to reduce the amount of fibers terminated in the COs. Figure 5b shows the PON architecture. That is a single fiber leaving the CO may support several NTs, depending on the splitting ratio. This architecture gives an important reduction in costs but also an increase of complexity. In this case, there is one fiber to be shared between all users and the amount of fiber is reduced.

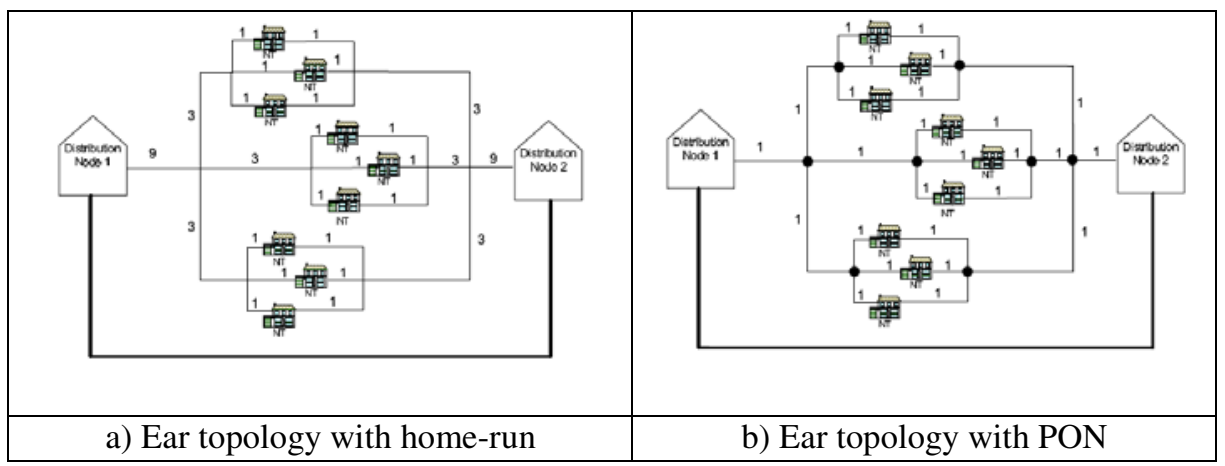

Fig. 5. Configuration for Ear topology 


\section{Case Study}

For the case study, the area of Hals is presented here. The Hals area is a small rural community in Denmark, with approximately 3500 private households and 11500 inhabitants spread over 191 square $\mathrm{km}$.

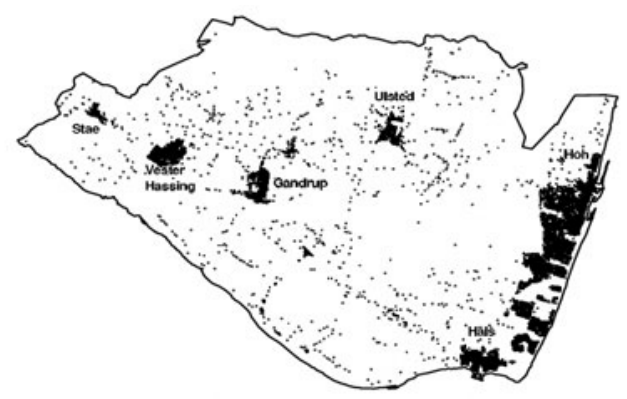

Fig. 6. Distribution of NTs in the Hals area

There are as much as 3400 summerhouses. The private households, public institutions and businesses are mainly situated in villages, while the agriculture is more spread out. Figure 6 shows the distribution of NTs in Hals area. The existing infrastructure is representative to the infrastructure in similar rural areas around the country. There are 7 central offices owned by the former national telecom, TDC. The existing central offices were chosen for the access network nodes. Only 5 ANs are decided that were located in rather big towns.

\section{Phases and Implementation Scenarios}

The study conducted the testing in two phases. The first phase focused on the dense local area and tested and compared several scenarios.. While the second phase focused on taking the results from phase one and applying the best solutions on a larger area, including rural areas.

\subsection{Phase One}

Basically there are three types of scenarios that are considered in this work, these are tree topology, ear topology and eartree topology. Further, each scenario was tested with HR and PON, and also with DN, in the same or different locations. This was done in a small focused area in Hals.

\subsubsection{Phase One Scenarios}

The scenarios were home run using the previous mentioned automated network planning and the GIS data. These scenarios are explained in table 1.

Figure 7a illustrates how the eartree topology was implemented with DNs in the same location, and adhering to the rules. Furthermore figure $7 \mathrm{~b}$ illustrates how the eartree topology is done with DN's on separate locations. 
Table 1. Scenarios and topologies

\begin{tabular}{|c|c|c|}
\hline \multirow{4}{*}{ 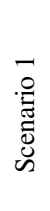 } & Scenario 1.HR.1 & Tree topology with home-run and 2DN in the same location \\
\hline & Scenario 1.HR.2 & Tree topology with home-run and 2DN in different location \\
\hline & Scenario 1.PON.1 & Tree topology with PON and 2DN in the same location \\
\hline & Scenario 1.PON.2 & Tree topology with PON and 2DN in different location \\
\hline \multirow{4}{*}{ 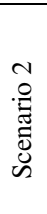 } & Scenario 2.HR.1 & Ear topology with home-run and 2DN in the same location \\
\hline & Scenario 2.HR.2 & Ear topology with home-run and 2DN in different location \\
\hline & Scenario 2.PON.1 & Ear topology with PON and 2DN in the same location \\
\hline & Scenario 2.PON.2 & Ear topology with PON and 2DN in different location \\
\hline \multirow{4}{*}{ 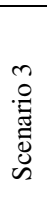 } & Scenario 3.HR.1 & Eartree topology with home-run and 2DN in the same location \\
\hline & Scenario 3.HR.2 & Eartree topology with home-run and 2DN in different location \\
\hline & Scenario 3.PON.1 & Eartree topology with PON and 2DN in the same location \\
\hline & Scenario 3.PON.2 & Eartree topology with PON and 2DN in different location \\
\hline
\end{tabular}

\subsubsection{Phase One Results}

The results obtained from the phase one - seen from Figure 8 - show that in comparison to the ear topology the amount of fiber is almost triple, making the cost difference about two and a half times more than in the tree topology. While a tree topology can place a splicing point (DN, or any kind of distribution point) in an optimal position to acquire the least amount of fiber. In order to offer the redundancy and offer savings on extra digging means the placement of the splicing points are faulty in this problem. Because it is necessary to situate them on each side of the district, the distance will grow more rapidly as we need to access NTs from each side and all over the area. This is actually noticed in the creation of the topology but as it was a theory, it is based on the fact that fiber prices are going down thus not making it a deciding factor. There are more important factors involved such as the blowing of the fiber.

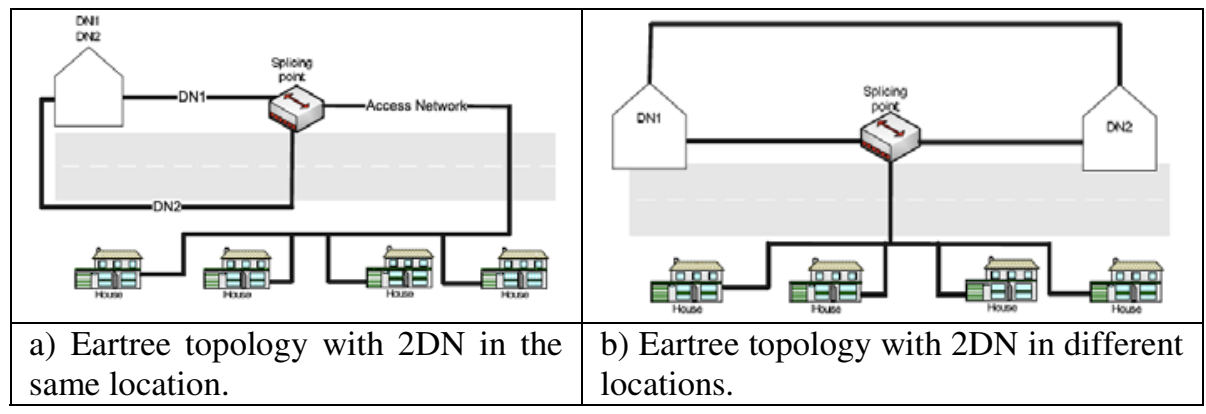

Fig. 7. Configuration of eartree topology 
All of this actually collaborated with the results acquired, making the ear topology expensive to deploy. The ear topology is still an interesting topology, if a method is found to decrease the fiber. Another solution is to look at it when the fiber prices have gone even further down, but then again, the tree topology cost would also decrease.

A new topology was introduced which could offer redundancy to the access network but not use an excessive amount of fiber like the ear topology. The result was the eartree topology, where each splicing point would be redundant. But from the splicing point the tree topology would be used. For this topology the absolute worst case scenario is that it would loose one district when either a splicing point would be made unavailable or a cut near the splicing point occured. The further the cut is from the splicing point, in most cases, the less the number of NTs that become affected. Now the only extra fiber needed is for doubling the main duct lines unaffecting the NT to splicing points fiber required. Now, this is done to limit the extra amount of fiber by not doubling the NT to SP. Comparing the cost it can be seen that the cost only increases around $25 \%$ between the eartree topology and tree topology throughout the scenarios - see Figure 8 - thus making eartree topology a feasible topology to investigate further in phase 2 .

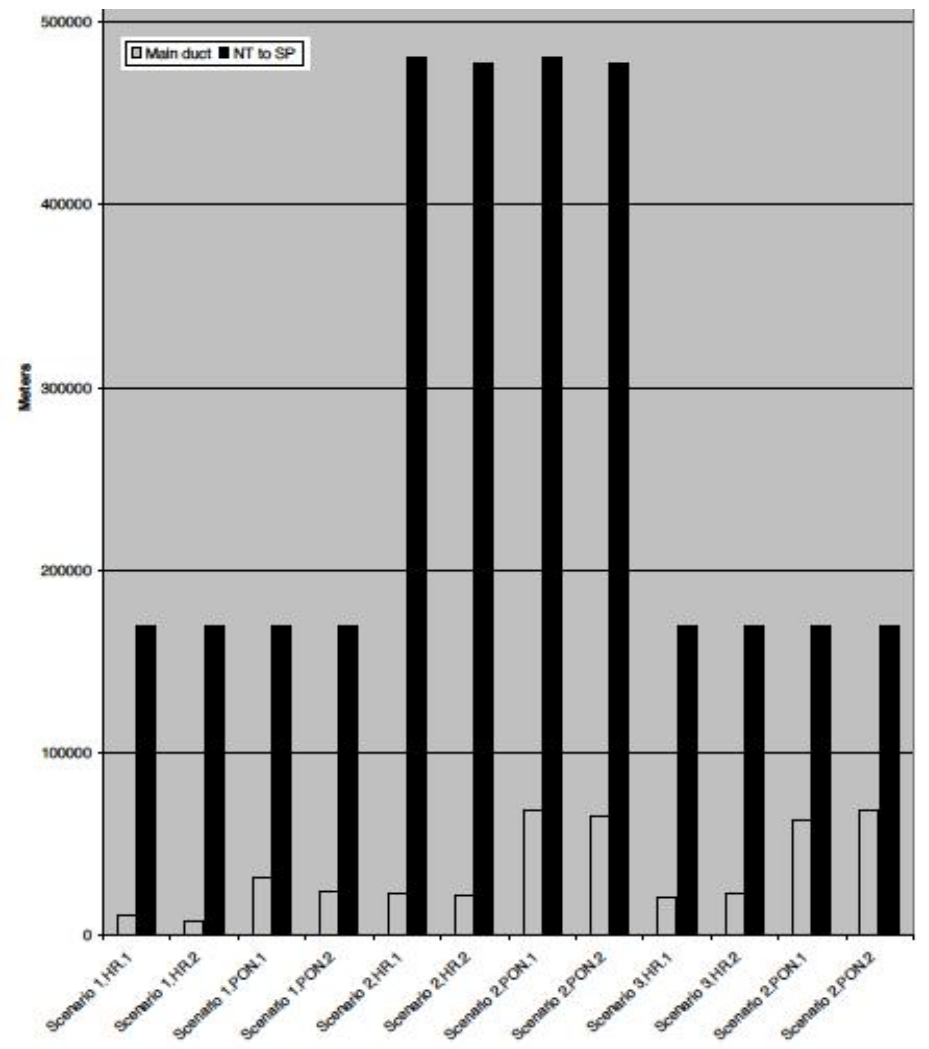

Fig. 8. Implementation results with amount of fibers 


\subsection{Phase Two}

Phase two focuses on using the best findings from phase one and putting them in a more real life scenario. Set of criteria's was done for phase two.

\subsubsection{Phase Two Criteria}

The criteria for phase two were created from phase one and are as follow:

1. Large scale testing

2. Investigate with automatic district creation effect on different sizes of districts

3. Two network scenarios

- Tree topology and ear topology

- Home-run only

- Distribution nodes in one location

Instead of using only one town in the municipality of Hals, the paper would now focus on the whole municipality, dense and rural areas. Several methods where tested for creating automatic districts, but the one chosen was a spanning tree algorithm starting from each DN. Placing of the splicing points was then done from the point with the shortest distance to all NTs.

\subsubsection{Phase Two Results}

In almost all cases smaller number of NTs per district shows decreased cost, this means that creating districts with only 30-40 NTs is the most feasible. This means that there is higher redundancy, where a single digging accident close to a splicing point will cause in the maximum 30-40 NTs to lose connection. Figure 9 illustrates the results from testing of size of districts in the ear topology.

Taking this result and applying the final cost for each topology shows only a $17 \%$ increase in cost between the use of tree topology and eartree topology. The results are very good and reinstate the fact that the eartree topology could be used as a viable

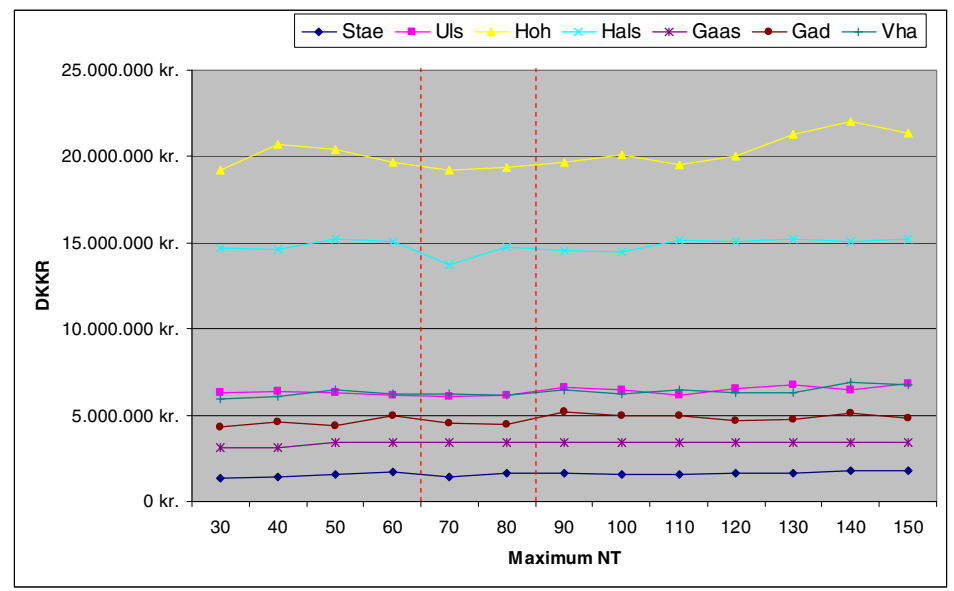

Fig. 9. District calculation result per town in the municipality 
solution for future network planning. Now with that much added redundancy to the access network and not that much increase in cost, the eartree topology is definitely a feasible/cheap solution to offer redundancy as close to NTs as possible.

\section{Conclusions}

The paper presents an implementation study of fiber access networks with and without redundancy by using an automated planning method. The motivation for doing is that networks are moving towards becoming a multi purpose. This means that more services for the home are now being offered through this network. In order to ensure reliability of these services some physical redundancy is needed. To date no practical investigations have been done into offering redundancy in the access network, meaning that it should be considered a necessity to review this.

The results showed that PON had little or no affect on the cost as the district method already mimicked the PON affect. Furthermore it showed little or no difference in the fiber usage when using two DN locations or one. In Phase one, three scenarios where created with the three topologies in question. The PON and DN location was included in those scenarios as sub scenarios, but as explained, had little effect. This project was unsuccessful in reducing the fiber enough for the ear topology to become a cost efficient solution. The eartree topology showed some good results, and in comparison to the tree topology, the cost difference of the fiber showed to be only around $25 \%$. Thus it was used in phase two.

In phase two the eartree topology was again compared to the tree topology, but now on a larger scale and focused with the criteria that showed best results. By looking at the district, and possibly making districts smaller and thus minimizing the need for single fiber, the cost could be reduced and the redundancy increased. This proved to be true as most areas showed that the sweet spot was 30-40 NTs per district. By using this, we were also able to reduce the difference in overall cost and the difference between the eartree topology and tree topology now only showed a $17 \%$ increase in cost, making this a highly feasible solution.

In conclusion, the results shown are quite good and are found to be a viable and interesting solution in offering redundancy or at least minimizing the affect of digging accidents to a few NTs. From this conclusion it is of interest to conduct further research on eartree topology in comparison to the tree topology in order to truly view whether redundant topology can be planned without costing more than a normal tree topology in a large scale environment.

\section{References}

1. Haraldsson, G.H., Beshir, O.: Automatic Network Planning for Access Networks in FTTH with and without Redundancy. Master thesis, Aalborg University (2007)

2. The Swedish ICT Commission. General guide to a future-proof IT infrastructure (April 2005) 
3. Pedersen, J.M., Riaz, T.M., Knudsen, T.P., Madsen, O.B.: Designing Broadband Access Network with triple redundancies. Department of Control Systems. Aalborg University (2005)

4. Haraldsson, G.H., Pedersen, J.M.: Cost vs. Redundancy in FTTH access networks: A case study of a Danish village. In: CSNDSP 2006, Patras, Greece (July 2006)

5. Lin, C. (ed.): Broadband Optical Access Networks and Fiber to the Home: Systems Technologies Development and Strategy. Wiley, Chichester (2006)

6. Pedersen, J.M.: Structural Quality of Service in Large-Scale Networks, PhD thesis, Department of Control Systems. Aalborg University (April 2005) 